\title{
Erratum: Polarized and resonant Raman spectroscopy on single InAs nanowires [Phys. Rev. B 84, 085318 (2011)]
}

\author{
M. Möller, M. M. de Lima Jr., A. Cantarero, L. C. O. Dacal, J. R. Madureira, F. Iikawa, T. Chiaramonte, and M. A. Cotta
}

(Received 6 June 2012; published 25 June 2012)

DOI: 10.1103/PhysRevB.85.239904

PACS number(s): 78.67.Uh, 78.30.Fs, 99.10.Cd

We found out that the polar pattern for the zinc-blende InAs LO mode displayed in Fig. 2(b) of our original paper represents the backscattering Raman intensities from a (112) top surface and not as stated in the original manuscript from a (110) top surface. In the latter the LO mode is forbidden for all configurations. In addition, varying the rotation angle $\theta$ with respect to the [112] direction (see Fig. 1 in the original paper) leads to changes in the polar patterns for the LO mode in case of perpendicular analysis, as illustrated in Fig. 1. For the parallel analyzer this mode is always forbidden. For completeness, the analytical expressions for the Raman intensities for both TO and LO modes as a function of the rotational angle $\theta$ and the polarization angle $\phi$ used to obtain the polar plots are given as

$$
\begin{gathered}
I_{\|}^{\mathrm{TO}}(\theta, \phi)=\frac{1}{6} d_{\mathrm{TO}}^{2}[5+3 \cos (2 \phi)], \\
I_{\perp}^{\mathrm{TO}}(\theta, \phi)=\frac{1}{6} d_{\mathrm{TO}}^{2}\{3-\cos (6 \theta)-2 \sin (3 \theta)[\cos (2 \phi) \sin (3 \theta)+\sqrt{2} \sin (2 \phi)]\}, \\
I_{\|}^{\mathrm{LO}}(\theta, \phi)=0, \\
I_{\perp}^{\mathrm{LO}}(\theta, \phi)=\frac{2}{3} d_{\mathrm{LO}}^{2} \cos ^{2}(3 \theta) \sin ^{2}(\phi) .
\end{gathered}
$$

These results do not affect the interpretation of the experiments on the wurtzite nanowires. In the case of the reference zinc-blende (110) InAs substrate, the appearance of the forbidden LO mode is attributed to the fact that the laser excitation energy $(2.41 \mathrm{eV})$ is close to the $E_{1}$ transition of InAs bulk $(2.57 \mathrm{eV})$, breaking the selection rules.

We would like to thank M. R. Correia for pointing out these discrepancies.

$$
\mathrm{LO}, \perp
$$

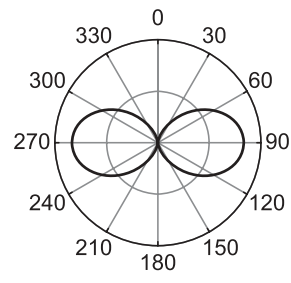

$\theta=0^{\circ}$

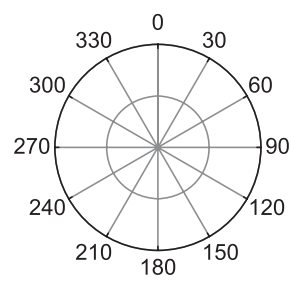

$\theta=30^{\circ}$

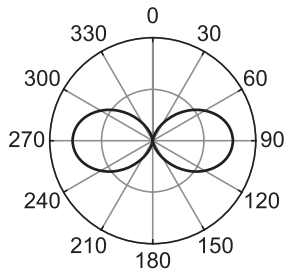

$\theta=05^{\circ}$

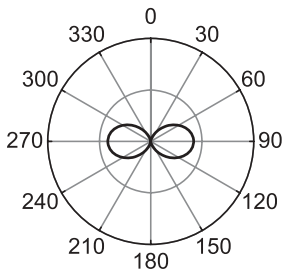

$\theta=45^{\circ}$

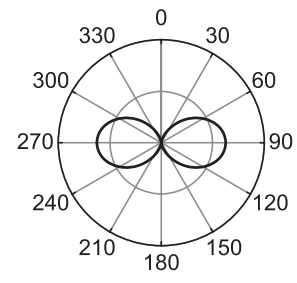

$\theta=10^{\circ}$

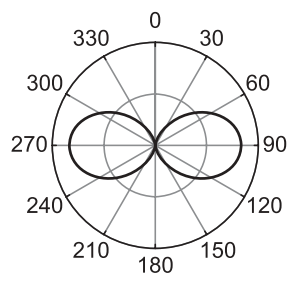

$\theta=60^{\circ}$

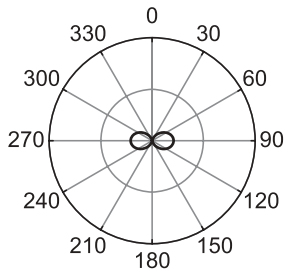

$\theta=20^{\circ}$

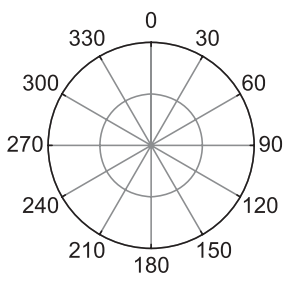

$\theta=90^{\circ}$

FIG. 1. Calculated intensity polar patterns of the LO mode under perpendicular analysis $(\perp)$ for different rotation angles $\theta$ for a zinc-blende nanowire oriented along the polarization angle $\phi=0^{\circ}$. The LO patterns have a periodicity of $60^{\circ}$ and the Raman signal vanishes for $\theta=30^{\circ}+N \times 60^{\circ}$, where $N$ is a integer. The maximum intensities are always obtained for the polarization angles $\phi=90^{\circ}, 270^{\circ}$. For $\theta=0^{\circ}$

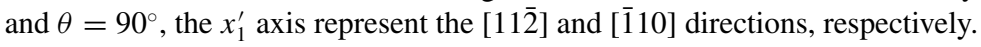

